\title{
Nuclear spin state narrowing via gate--controlled Rabi oscillations in a double quantum dot
}

\author{
D. Klauser, W. A. Coish, and Daniel Loss \\ Department of Physics and Astronomy, University of Basel, \\ Klingelbergstrasse 82, CH-4056 Basel, Switzerland
}

(Dated: July 17, 2018)

\begin{abstract}
We study spin dynamics for two electrons confined to a double quantum dot under the influence of an oscillating exchange interaction. This leads to driven Rabi oscillations between the $|\uparrow \downarrow\rangle$-state and the $|\downarrow \uparrow\rangle$-state of the two-electron system. The width of the Rabi resonance is proportional to the amplitude of the oscillating exchange. A measurement of the Rabi resonance allows one to narrow the distribution of nuclear spin states and thereby to prolong the spin decoherence time. Further, we study decoherence of the two-electron states due to the hyperfine interaction and give requirements on the parameters of the system in order to initialize in the $|\uparrow \downarrow\rangle$-state and to perform a $\sqrt{\text { SWAP }}$ operation with unit fidelity.

PACS numbers: 73.21.La,76.20.+q,76.30.-v,85.35.Be
\end{abstract}

\section{INTRODUCTION}

One of the important proposals for quantum information processing in solid-state systems is the spinqubit proposal for quantum computing with electron spins in quantum dots 1 . Much effort has been put into the realization of this proposal leading to exciting theoretical ${ }^{2}$ and experimental achievements $\frac{3,4,5.6 .7 .8 .9}{2}$ Still many challenges remain such as decoherence and the implementation of single-qubit gates.

A major obstacle to quantum computation with the quantum-dot spin qubit is decoherence due to the coupling of the qubit to its environment. The hyperfine interaction between the electron spin and the nuclear spins present in all III-V semiconductors 10 leads to the strongest decoherence effect $9.11,12,13,14.15,16.17 .18$. Experiments $8.9,19,20$ have yielded values for the freeinduction spin dephasing time $T_{2}^{*}$ that are consistent with $T_{2}^{*} \sim \sqrt{N} / A \sim 10 \mathrm{~ns}^{14.15 .16}$ for $N=10^{6}$ and $A=90 \mu \mathrm{eV}$ in GaAs, where $N$ is the number of nuclei within one quantum dot Bohr radius and $A$ characterizes the hyperfine coupling strength ${ }^{21}$ This is to be contrasted to potential spin-echo envelope decay, which may be much larger $22,23,24$ With a two-qubit switching time of $\tau_{s} \sim 50 \mathrm{ps} 11$ this only allows $\sim 10^{2}$ gate operations within $T_{2}^{*}$, which falls short (by a factor of 10 to $10^{2}$ ) of current requirements for efficient quantum error correction ${ }^{25}$

There are several ways to overcome the problem of hyperfine-induced decoherence, of which measurement and thus projection of the nuclear spin state seems to be the most promising one $\frac{17}{}$. Other methods include polarization 11,16.17.26 of the nuclear spins and spin echo techniques $\frac{9.17 .23}{3}$ However, in order to extend the decay time by an order of magnitude through polarization of the nuclear spins, a polarization of above $99 \%$ is required, ${ }^{17}$ but the best result so far reached is only $\sim 60 \%$ in quantum dots 3.19 With spin-echo techniques, gate operations still must be performed within the single- spin free-induction decay time, which requires faster gate operations. A projective measurement of the nuclear spin state leads to an extension of the free-induction decay time for the spin. This extension is only limited by the ability to do a strong measurement since the longitudinal nuclear spin in a quantum dot is expected to survive up to the spin diffusion time, which is on the order of seconds for nuclear spins surrounding donors in GaAs ${ }_{27}$

The implementation of quantum computation schemes requires coherent control of the qubits. Rabi oscillations between the two qubit states are an important signature of coherence and thus observation of controlled Rabi oscillations is an important intermediate step in the experimental implementation of quantum information processors. Despite recent experimental achievements $\stackrel{3.9}{\underline{.} \text { there }}$ has still been no experimental observation of driven Rabi oscillations for a system of two quantum-dot spin qubits. What has been observed is electron spin resonance via $g$ tensor modulation in a bulk semiconductor 28

In the quantum-dot spin qubit proposal, two-qubit gates are realized through tuning of the exchange coupling $J$ between the two spins $\stackrel{1.11}{=}$ The splitting between singlet and triplet states of the two-electron system is given by the exchange coupling $J$ and in devices such as those in Refs. 9 and $8, J$ can be controlled through gate voltages. Petta et $a l^{\frac{9}{}}$ have recently managed to implement the $\sqrt{\mathrm{SWAP}}$-gate in their setup. However, in order to implement single-qubit gates, control over local magnetic fields or $\mathrm{g}$-factors is required $\underline{\underline{11}}$

As we will show in Sec [1 an oscillating exchange $J(t)$ induces Rabi oscillations between the states $|\uparrow \downarrow\rangle$ and $|\downarrow \uparrow\rangle$ of two electron spins (one electron in each dot). The amplitude of these oscillations is resonant on the splitting between $|\uparrow \downarrow\rangle$ and $|\downarrow \uparrow\rangle$ and the width of this resonance is proportional to the amplitude $j$ of the oscillating component of $J(t)=J_{0}+j \cos (\omega t)$, where $\omega$ is the driving frequency. Since the splitting depends on the state of the nuclear system, a measurement of the resonance is also a measurement of the state of the nuclear spins and thus provides a way to narrow the quantum distribu- 
tion of the nuclear spin states. This narrowing of the spin state is one possible solution to suppress hyperfineinduced decoherence in quantum-dot spin qubits 17 . It has been proposed to measure the nuclear spin polarization using a phase estimation method ${ }^{29}$ In the ideal case, phase estimation yields one bit of information about the nuclear-spin system for each perfectly measured electron. Optical methods have also been proposed ${ }^{30}$ The all-electrical method we present here can be applied with current technology.

The rest of this paper is organized as follows. In Sec. III we show that an oscillating exchange leads to driven Rabi oscillations and calculate the resonance linewidth. In Sec. III) we propose a method to narrow the distribution of the nuclear spin states. in Sec. IV we consider decoherence induced through the hyperfine interaction for a static exchange coupling $J$. We use these results in Sec $\nabla$ to analyze under which conditions we reach unit fidelity for the initialization to the state $|\uparrow \downarrow\rangle$ and a $\sqrt{\text { SWAP }}$ operation $\frac{1}{1}$ Sec. VI contains a summary of our results.

\section{OSCILLATING EXCHANGE AND ESR}

In this section we show that under suitable conditions an oscillating exchange interaction may be used to induce Rabi oscillations in a system of two electrons confined to a double quantum dot like those in Refs. 6.789 .

We denote by $\mathbf{h}_{i}=\left(h_{i}^{x}, h_{i}^{y}, h_{i}^{z}\right), i=1,2$, the collective quantum nuclear spin operator, the "Overhauser operator", in dot one and two, respectively, and write $\delta h^{z}=\frac{1}{2}\left(h_{1}^{z}-h_{2}^{z}\right)$. The collective quantum nuclear spin operator $\mathbf{h}_{i}$ is defined as $\mathbf{h}_{i}=\sum_{k} A_{k}^{i} \mathbf{I}_{k}$, where $\mathbf{I}_{k}$ is the nuclear spin operator for a nucleus of total spin $I$ at lattice site $k$, and the hyperfine coupling constants are given by $A_{k}^{i}=v A\left|\psi_{0}^{i}\left(\mathbf{r}_{k}\right)\right|^{2}$, where $v$ is the volume of a unit cell containing one nuclear spin, A characterizes the hyperfine coupling strength, and $\psi_{0}^{i}\left(\mathbf{r}_{k}\right)$ is the single-particle envelope wavefunction of the electron evaluated at site $k$. Further, $\langle\mathcal{O}\rangle_{\mathrm{rms}}=\left\langle\psi_{I}\left|\mathcal{O}^{2}\right| \psi_{I}\right\rangle^{1 / 2}$ is the root-meansquare expectation value of the operator $\mathcal{O}$ with respect to the nuclear spin state $\left|\psi_{I}\right\rangle$. We assume that the Zeeman splitting $\epsilon_{z}=g \mu_{B} B$ induced by a uniform applied magnetic field $\mathbf{B}=(0,0, B), B>0$, is much larger than $\langle\delta \mathbf{h}\rangle_{\mathrm{rms}}$ and $\left\langle\mathbf{h}_{i}\right\rangle_{\mathrm{rms}}$. Under these conditions the relevant spin Hamiltonian becomes block diagonal with blocks labeled by the total electron spin projection along the magnetic field $S^{z}$. In the subspace of $S^{z}=0$ the Hamiltonian can be written as $(\hbar=1)^{18}$

$$
H_{0}=\frac{J}{2}\left(1+\tau^{z}\right)+\delta h^{z} \tau^{x}+\delta b^{z} \tau^{x} .
$$

Here, $J$ is the Heisenberg exchange coupling between electron spins on the two dots and $\delta b^{z}$ the inhomogeneity of an externally applied classical static magnetic field which we add in addition to the treatment in Ref. 18. Further, $\tau=\left(\tau^{x}, \tau^{y}, \tau^{z}\right)$ is the vector of Pauli matrices in the basis of $S^{z}=0$ singlet $|S\rangle$ and triplet $\left|T_{0}\right\rangle$
$\left(|S\rangle \rightarrow\left|\tau^{z}=-1\right\rangle,\left|T_{0}\right\rangle \rightarrow\left|\tau^{z}=+1\right\rangle\right)$. It has been proposed to use two pseudo-spin states such as $|S\rangle$ and $\left|T_{0}\right\rangle$ as a logical qubit 31

We assume a time-dependent exchange of the form

$$
J=J(t)=J_{0}+j \cos (\omega t) .
$$

The operator $\delta h^{z}$ commutes with the Hamiltonian at all times. Thus, if the nuclear-spin system is in an eigenstate $|n\rangle$ of $\delta h^{z}$ with $\delta h^{z}|n\rangle=\delta h_{n}^{z}|n\rangle$, we have $H|\psi\rangle=$ $H_{n}\left|\psi_{e}\right\rangle \otimes|n\rangle$, where in $H_{n}$ the operator $\delta h^{z}$ has been replaced by $\delta h_{n}^{z}$ and $\left|\psi_{e}\right\rangle$ is the electron spin part of the wave function. In order to bring $H_{n}$ to a form that is very similar to the standard ESR (electron spin resonance) Hamiltonian $^{32}\left(H_{E S R}=-\frac{1}{2} \epsilon_{z} \sigma_{z}-\frac{1}{2} \Delta_{x} \cos (\omega t) \sigma_{x}\right)$ we perform a unitary transformation $U_{1}=\exp \left(-i \frac{\pi}{4} \tau^{y}\right)$ which is just a rotation about the $y$-axis in a Blochsphere picture. Also introducing $\Omega_{n}=2\left(\delta h_{n}^{z}+\delta b^{z}\right)$, the above Hamiltonian becomes

$$
\tilde{H}_{n}=U_{1} H_{n} U_{1}^{\dagger}=\frac{J_{0}}{2} \tau^{x}+\frac{j}{2} \cos (\omega t) \tau^{x}-\frac{1}{2} \Omega_{n} \tau^{z} .
$$

The Pauli matrices are now given in the new basis of $|\downarrow \uparrow\rangle=\left|\tau^{z}=1\right\rangle=|+\rangle$ and $|\uparrow \downarrow\rangle=\left|\tau^{z}=-1\right\rangle=|-\rangle$. For $J_{0}=0$ this is just the standard ESR Hamiltonian. We have evaluated pseudo-spin dynamics under this Hamiltonian in a rotating wave approximation close to resonance for $j \ll \Omega_{n}$. When we treat the $J_{0}$-term as a perturbation and calculate the transition probability between unperturbed eigenstates of the Hamiltonian we find that it is proportional to $J_{0}^{2} / \Omega_{n}^{2}$ and we may thus neglect this term close to resonance and if $J_{0} \ll \Omega_{n}$. Hence, we are left with the standard ESR Hamiltonian which leads to Rabi oscillations. Initializing the two-electron system in the state $|\downarrow \uparrow\rangle=|+\rangle$ (which can be done as proposed in Sec. $\nabla$ we obtain for the expectation value of $\tau^{z}(t)$ :

$$
\begin{aligned}
\left\langle\tau^{z}(t)\right\rangle_{n}= & \left\langle n\left|\otimes\left\langle+\left|\tau^{z}(t)\right|+\right\rangle \otimes\right| n\right\rangle \\
= & \frac{\left(\Omega_{n}-\omega\right)^{2}+(j / 2)^{2} \cos \left(\omega^{\prime} t\right)}{\left(\Omega_{n}-\omega\right)^{2}+(j / 2)^{2}}, \\
& \omega^{\prime}=2 \sqrt{\left(\Omega_{n}-\omega\right)^{2}+(j / 2)^{2}}, \\
& j \ll \Omega_{n}, \quad J_{0} \ll \Omega_{n},\left|\Omega_{n}-\omega\right| \ll \Omega_{n} .
\end{aligned}
$$

For $\omega=\Omega_{n}$ the system undergoes coherent Rabi oscillations between the states $|+\rangle$ and $|-\rangle$ with a frequency of $j$. Averaged over time, the expectation value of $\tau^{z}$ is

$$
\left\langle\left\langle\tau^{z}\right\rangle_{n}\right\rangle=\lim _{T \rightarrow \infty} \frac{1}{T} \int_{0}^{T}\left\langle\tau^{z}(t)\right\rangle_{n} d t=\frac{\left(\Omega_{n}-\omega\right)^{2}}{\left(\Omega_{n}-\omega\right)^{2}+(j / 2)^{2}} .
$$

In order to measure the time-averaged value $\left\langle\left\langle\tau^{z}\right\rangle_{n}\right\rangle$ the measurement time must be much larger than the period of Rabi oscillations ( $\sim 1 / j$ on resonance). $1-\left\langle\left\langle\tau^{z}\right\rangle_{n}\right\rangle$ has a Lorentzian lineshape with a full width at half maximum (FWHM) of $j$. Most importantly, the resonance frequency depends on the nuclear-spin eigenstate through $\Omega_{n}=2\left(\delta h_{n}^{z}+\delta b^{z}\right)$ and thus a measurement of the resonance will determine $\delta h_{n}^{z}$. 


\section{A. Superposition of nuclear-spin eigenstates}

Before a measurement on the nuclear-spin system is performed, there is no reason for the nuclear-spin system to be in an eigenstate of $\delta h^{z}$, but it is most likely in some generic superposition of these eigenstates. Thus, we now investigate how the resonance changes if we consider the nuclear-spin system to be in a superposition of eigenstates of the collective nuclear spin operator $\delta h^{z}$.

At $t=0$ we fix the electron system in the state $|\downarrow \uparrow\rangle=$ $|+\rangle$ while the nuclear-spin system is in an arbitrary state: $\rho(0)=\rho_{e}(0) \otimes \rho_{I}(0)$ with

$$
\begin{aligned}
& \rho_{e}(0)=|+\rangle\langle+|, \\
& \rho_{I}(0)=\sum_{i} p_{i}\left|\psi_{I}^{i}\right\rangle\left\langle\psi_{I}^{i}|;| \psi_{I}^{i}\right\rangle=\sum_{n} a_{n}^{i}|n\rangle,
\end{aligned}
$$

where the $a_{n}^{i}$ satisfy the normalization condition $\sum_{n}\left|a_{n}^{i}\right|^{2}=1$ and $\sum_{i} p_{i}=1$. Here, $\rho_{I}(n)=\sum_{i} p_{i}\left|a_{n}^{i}\right|^{2}$ are the diagonal elements of the nuclear-spin density operator. The Hamiltonian $H_{0}$ commutes with $\delta h^{z}$ and thus we find

$$
\overline{\left\langle\tau^{z}(t)\right\rangle}=\sum_{n} \rho_{I}(n)\left\langle\tau^{z}(t)\right\rangle_{n}
$$

which defines the overbar.

We assume that for a large number of nuclear spins $N \gg 1$ which are in a superposition of $\delta h^{z}$-eigenstates $|n\rangle, \rho_{I}(n)$ describes a continuous Gaussian distribution of $\delta h_{n}^{z}$ values, with mean $\overline{\delta h^{z}}$ and variance $\sigma^{2}=$ $\overline{\left(\delta h^{z}-\overline{\delta h^{z}}\right)^{2}}$. In the limit of large $N$ the approach to a Gaussian distribution for a sufficiently randomized nuclear system is guaranteed by the central limit theorem 17 We perform the continuum limit according to

$$
\begin{aligned}
\sum_{n} \rho_{I}(n) f(n) & \rightarrow \int d x \rho_{I ; \bar{x}, \sigma}(x) f(x), \\
\rho_{I ; \bar{x}, \sigma}(x) & =\frac{1}{\sqrt{2 \pi} \sigma} \exp \left(-\frac{(x-\bar{x})^{2}}{2 \sigma^{2}}\right),
\end{aligned}
$$

where $x=\delta h_{n}^{z}, \bar{x}=\overline{\delta h^{z}}$ and $\sigma^{2}=\overline{x^{2}}-\bar{x}^{2}$. The only effect of $\delta b^{z}$ is to shift the mean value of the Overhauser field inhomogeneity to $x_{0}=\bar{x}+\delta b^{z}$, whereas the width is left unchanged: $\sigma_{0}=\sigma$. According to this description we obtain

$$
\begin{aligned}
\overline{\left\langle\tau^{z}(t)\right\rangle} & =\int_{-\infty}^{\infty} d x \rho_{I ; x_{0}, \sigma_{0}}(x)(f(x)+g(x, t)) \\
f(x) & =\frac{(2 x-\omega)^{2}}{(2 x-\omega)^{2}+(j / 2)^{2}} \\
g(x, t) & =\frac{(j / 2)^{2} \cos \left(2 \sqrt{(2 x-\omega)^{2}+(j / 2)^{2}} t\right)}{(2 x-\omega)^{2}+(j / 2)^{2}}
\end{aligned}
$$

The second term (Eq. (15) vanishes when it is averaged over time and we find

$$
1-\left\langle\overline{\left\langle\tau^{z}\right\rangle}\right\rangle=\frac{1}{2 \sigma_{0} \sqrt{2 \pi}} \int_{-\infty}^{\infty} d x \exp \left(-\frac{\left(x-2 x_{0}\right)^{2}}{8 \sigma_{0}^{2}}\right) \frac{(j / 2)^{2}}{(x-\omega)^{2}+(j / 2)^{2}}
$$

This integral (a convolution of a Lorentzian and Gaussian) is the well-known Voigt function, 33 and the resulting lineshape is the so-called "Voigt profile". The Voigt function may be expressed as $\left(\tilde{\omega}=j+4 i x_{0}-2 i \omega\right)$

$$
\left\langle\overline{\left\langle\tau^{z}\right\rangle}\right\rangle=1-\frac{j}{4 \sigma_{0}} \sqrt{\frac{\pi}{2}} \operatorname{Re}\left[\exp \left(\frac{\tilde{\omega}^{2}}{32 \sigma_{0}^{2}}\right) \operatorname{erfc}\left(\frac{\tilde{\omega}}{4 \sqrt{2} \sigma_{0}}\right)\right],
$$

where $\operatorname{erfc}(z)$ is the complementary error function. In the regime where $\sigma_{0} \ll j$ we may approximate the Lorentzian in the convolution (Eq. (16) by its value at $x=2 x_{0}$ and obtain

$$
\left\langle\overline{\left\langle\tau^{z}\right\rangle}\right\rangle \approx \frac{\left(2 x_{0}-\omega\right)^{2}}{\left(2 x_{0}-\omega\right)^{2}+(j / 2)^{2}} ; \quad \sigma_{0} \ll j .
$$

In this case the resulting resonance has the same FWHM as the Lorentzian, viz. $j$. On the other hand, if $\sigma_{0} \gg j$, we may approximate the Gaussian with its value at $x=\omega$ and thus obtain

$$
\left\langle\overline{\left\langle\tau^{z}\right\rangle}\right\rangle \approx 1-\frac{j}{4 \sigma_{0}} \sqrt{\frac{\pi}{2}} \exp \left(-\frac{\left(2 x_{0}-\omega\right)^{2}}{8 \sigma_{0}^{2}}\right) ; \quad \sigma_{0} \gg j .
$$

In this regime the width is twice the width $\sigma_{0}$ of the Gaussian distribution of the nuclear spin states. In order to make a statement about the width of the Voigt profile in general we look at the peak-to-peak separation $\Delta_{V}$ of the first derivative of the Voigt profile. For a Gaussian with a standard deviation of $2 \sigma_{0}$ we find $\Delta_{G}=4 \sigma_{0}$ for the peak-to-peak separation of the derivative and for a Lorentzian with FWHM of $j$ we have $\Delta_{L}=j / \sqrt{3}$. A Padé approximant for $\Delta_{V}$ in terms of $\Delta_{L}$ and $\Delta_{G}$ yields ${ }^{34}$

$$
\Delta_{V}=\frac{\Delta_{G}^{2}+a_{1} \Delta_{G} \Delta_{L}+a_{2} \Delta_{L}^{2}}{\Delta_{G}+a_{2} \Delta_{L}}
$$


where $a_{1}=0.9085, a_{2}=0.4621$. This approximation is accurate to better than $0.01 \Delta_{V}$ for all values

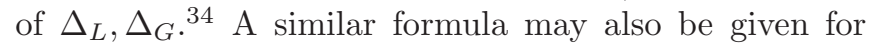
the half width at half maximum (HWHM) of the Voigt profile ${ }^{35}$

\section{STATE NARROWING}

The general idea behind state narrowing is that the evolution of the two-electron system is dependent on the nuclear spin state and thus knowing the evolution of the two-electron system determines the nuclear spin state. Thus, in this section we describe how the Gaussian superposition $\rho_{I ; \sigma_{0}, x_{0}}(x)$ of collective nuclear spin eigenstates $|n\rangle$ can be narrowed through a sequence of measurements performed on a double quantum dot on a time scale much less than the timescale of variation of $\delta h^{z}$ and for $j \lesssim \sigma_{0}$. We first give a general description of how a complete measurement of the lineshape of the Rabi resonance narrows the Gaussian superposition. Such a complete measurement of the lineshape consists of many single measurements of the operator $\tau^{z}$. In Sec. IIIA we present a detailed analysis of such a complete measurement and in $\mathrm{Sec}$ ЩIB we discuss different measurement schemes.

The operator $\delta h^{z}$ was defined in Sec. II and it describes the difference in the z-components of total nuclear field in each of the two dots. The total nuclear field is the result of $N \sim 10^{6}$ single nuclear spins and thus the eigenvalues of $\delta h^{z}$ will be highly degenerate. In the limit of large $N$ the spectrum of $\delta h^{z}$ is quasi-continuous and the probability density of eigenvalues of $\delta h^{z}$ is given by a Gaussian distribution, as described in Sec. ПIA For such a Gaussian superposition of nuclear spin eigenstates, the lineshape of the Rabi resonance is given by a Voigt profile, as described in Sec. [A This Voigt profile can be seen as a superposition of Lorentzian lineshapes, where each Lorentzian results from a nuclear spin eigenvalue $\delta h_{n}^{z}$ and is centered around $\Omega_{n}=2\left(\delta h_{n}^{z}+\delta b^{z}\right)$. In the Voigt profile, these Lorentzian lineshapes are weighted according to the amplitude of the corresponding eigenvalue $\delta h_{n}^{z}$ in the Gaussian-distributed superposition. Through a perfect complete measurement of the Rabi-resonance lineshape, the superposition of Lorentzian lineshapes collapses and we are left with one single Lorentzian (see figure (1). This Lorentzian corresponds to one single eigenvalue of $\delta h^{z}$ and thus the Gaussian distribution has been narrowed to zero width; the nuclear-spin system is in a state with fixed eigenvalue $\delta h_{n}^{z}$.

In principle, we would need to do infinitely many single measurements in order to completely measure the lineshape of the Rabi resonance with perfect accuracy, since each point on this resonance curve is a (time-averaged) expectation value of the quantum mechanical operator $\tau^{z}$. Still, we may perform a finite number $M$ of single measurements (see Sec. IIIA) for each of a set of driving frequencies $\omega$ and thus obtain the series of expectation values for different $\omega$ up to some error. This error de-
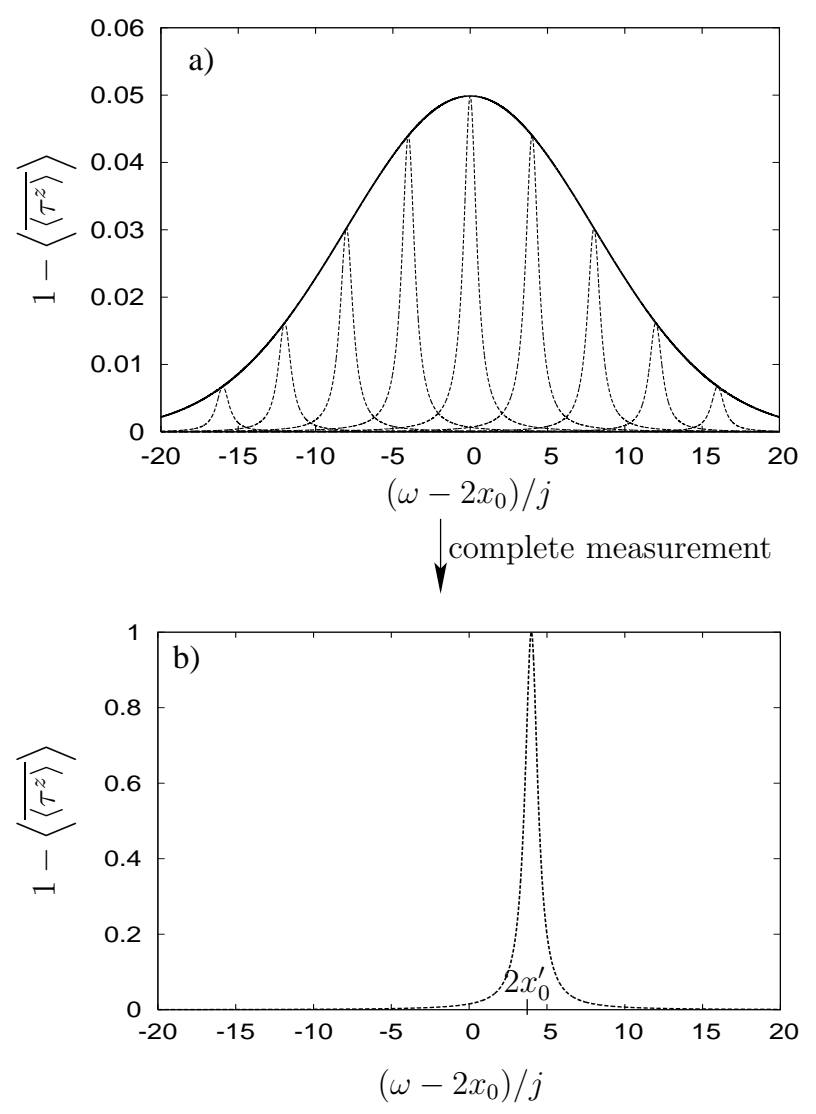

FIG. 1: a) This figure illustrates the projection obtained through an ideal complete measurement of the Rabiresonance lineshape. All the different Lorentzian resonances corresponding to different nuclear spin eigenstates add up to a Gaussian lineshape. b) Through a perfect complete measurement of the lineshape of the Rabi resonance, which involves many single measurements of $\tau^{z}$, the superposition collapses and we are left with one single Lorentzian centered around $2 x_{0}^{\prime}=\Omega_{n}$, which in general is different from $2 x_{0}$.

pends on $M$. There will then in general be more than one Lorentzian which can be fit (within error) to these expectation values and thus we would not narrow to zero width. We would still have a distribution of nuclear spin eigenstates, but one with smaller width than before the measurements.

For such a narrowing through measurement to be successful, the amplitude $j$ of the oscillating exchange $J(t)$ which determines the width of the Lorentzian lineshapes should be smaller than the width $\sigma_{0}$ of the Gaussian distribution. Otherwise, the Rabi resonance would be dominated by the Lorentzian (see Eq. (18) ) and the method would not result in narrowing of the nuclear-spin distribution. The general requirements on the system parameters to narrow the distribution of nuclear spin eigenvalues are

$$
j, J_{0}, \sigma_{0} \ll x_{0} ; \quad j \lesssim \sigma_{0}
$$


We note that, unlike in standard ESR, power absorption is not measured here, but instead the expectation value of the pseudo-spin $\tau^{z}$, for instance via a quantum point contact (QPC) nearby one quantum dot (for a detailed description of the measurement process via such a QPC we refer the interested reader to Ref. 36). To determine the expectation value of the pseudo-spin $\tau^{z}$ many single measurements of the pseudo-spin are necessary and we thus proceed to give a detailed description of the state narrowing by considering the effect of these single measurements on the nuclear spin state.

\section{A. Description of state narrowing by consecutive pseudo-spin measurements}

In this subsection we describe in detail how a single measurement of the pseudo-spin $\tau^{z}$ of the two-electron system affects the nuclear-spin system. Further, we give a general formula for the diagonal elements of the nuclear-spin-system density operator in the continuum limit after $M$ measurements. The sequence of $M$ measurements is referred to as a "complete measurement".

At $t=0$ the two-electron system is initialized to the state $|+\rangle=|\downarrow \uparrow\rangle$ and we assume that the electron and the nuclear system are initially factorized. Thus, the total system at $t=0$ is described generally by the following density operator

$$
\rho(0)=\rho_{e}(0) \otimes \rho_{I}(0)=|+\rangle\left\langle+\left|\otimes \sum_{i} p_{i}\right| \psi_{I}^{i}\right\rangle\left\langle\psi_{I}^{i}\right|,
$$

with nuclear-spin state $\left|\psi_{I}^{i}\right\rangle=\sum_{n} a_{n}^{i}|n\rangle$. The diagonal elements of the nuclear-spin density operator at $t=0$ are given by $\rho_{I}(n)=\rho_{I}(n, 0)=\sum_{i} p_{i}\left|a_{n}^{i}\right|^{2}$ and in the continuum limit we obtain the probability density $\rho_{I ; \bar{x}, \sigma}(x)$ for the eigenvalues $\delta h_{n}^{z}=x$ as given in Eq.(12). At time $t_{m}$ a measurement of the two-electron system (at driving frequency $\omega$, where $\omega$ is defined in Eq.(2) ) is performed with two possible outcomes $|+\rangle$ and $|-\rangle$. The diagonal elements of the nuclear-spin density operator after the measurement are given by (see Appendix B)

$$
\rho_{I}^{(1, \pm)}\left(n, t_{m}\right)=\frac{\rho_{I}(n, 0)}{P^{ \pm}\left(t_{m}\right)} \frac{1}{2}\left(1 \pm\left\langle\tau^{z}\left(t_{m}\right)\right\rangle_{n}\right),
$$

where $\left\langle\tau^{z}(t)\right\rangle_{n}$ is given by Eq. (4) and the probabilities $P^{ \pm}\left(t_{m}\right)$ to measure $| \pm\rangle$ are

$$
P^{ \pm}\left(t_{m}\right)=\sum_{i} \sum_{n} \frac{1}{2}\left(1 \pm\left\langle\tau^{z}\left(t_{m}\right)\right\rangle_{n}\right) p_{i}\left|a_{n}^{i}\right|^{2} .
$$

In the case where a measurement is performed with a low time resolution $37 \Delta t$, i.e., if $\Delta t \gg 1 / j$, the density operator after the measurement is the time average over the time interval $\Delta t$ and the cosine term in $\left\langle\tau^{z}\left(t_{m}\right)\right\rangle_{n}$ averages out (note that in the case of a measurement with low time resolution, $t_{m}$ is arbitrary, as long as $\Delta t$ is chosen to be large enough). For the rest of this subsection we thus assume $\frac{38}{38}$ that measurements are performed with low time resolution $\Delta t \gg 1 / j$. Further, we perform the continuum limit and obtain for the probability density of eigenvalues, i.e., the diagonal part of the density operator in the continuum limit (with $x=\delta h_{n}^{z}+\delta b^{z}$ and $\rho_{I}(x) \equiv$ $\rho_{I ; x_{0}, \sigma_{0}}(x)$, see Eq.(12)):

$$
\begin{aligned}
& \rho_{I}^{(1,+, \omega)}(x)=\rho_{I}(x)\left(1-L_{\omega}(x)\right) \frac{1}{P_{\omega}^{+}} \\
& \rho_{I}^{(1,-, \omega)}(x)=\rho_{I}(x) L_{\omega}(x) \frac{1}{P_{\omega}^{-}}
\end{aligned}
$$

where the probabilities for measuring $|+\rangle$ or $|-\rangle$ are given by

$$
\begin{aligned}
& P_{\omega}^{+}=\int_{-\infty}^{\infty} d x \rho_{I}(x)\left(1-L_{\omega}(x)\right) \\
& P_{\omega}^{-}=\int_{-\infty}^{\infty} d x \rho_{I}(x) L_{\omega}(x)
\end{aligned}
$$

with

$$
L_{\omega}(x)=\frac{1}{2} \frac{(j / 4)^{2}}{\left(x-\frac{\omega}{2}\right)^{2}+(j / 4)^{2}} .
$$

After the first measurement, the two-electron system is reinitialized to the state $|+\rangle$ if necessary and a second measurement is performed. Since the initial density matrix factors out in the above results, it is clear how to generalize Eqs. 25) and (26) to the case where $M$ consecutive measurements (without randomization of the nuclear-spin system in between measurements) are performed: every time $|+\rangle$ is measured, the diagonal elements $\rho_{I}(x)$ of the nuclear density matrix is multiplied by $1-L_{\omega}(x)$ and every time $|-\rangle$ is measured, $\rho_{I}(x)$ is multiplied by $L_{\omega}(x)$. Thus, we obtain the diagonal elements $\rho_{I}^{\left(M, \alpha^{-}, \omega\right)}(x)$ of the nuclear density matrix after $M$ measurements, of which $\alpha^{-}$times the measurement outcome was $|-\rangle$ (and $\left(M-\alpha^{-}\right)$-times $\left.|+\rangle\right)$:

$$
\rho_{I}^{\left(M, \alpha^{-}, \omega\right)}(x)=\frac{\rho_{I}(x)}{Q_{\omega}\left(M, \alpha^{-}\right)} W_{\omega}\left(M, \alpha^{-} ; x\right) .
$$

Here, $W_{\omega}\left(M, \alpha^{-} ; x\right)$ and the normalization factor $Q_{\omega}\left(M, \alpha^{-}\right)$are given by

$$
\begin{aligned}
W_{\omega}\left(M, \alpha^{-} ; x\right) & =L_{\omega}(x)^{\alpha^{-}}\left(1-L_{\omega}(x)\right)^{M-\alpha^{-}} \\
Q_{\omega}\left(M, \alpha^{-}\right) & =\int_{-\infty}^{\infty} d x \rho_{I}(x) W_{\omega}\left(M, \alpha^{-} ; x\right) .
\end{aligned}
$$

The normalization factor $Q_{\omega}\left(M, \alpha^{-}\right)$is related to $P_{\omega}^{ \pm}$ through $P_{\omega}^{-}=Q_{\omega}(1,1), P_{\omega}^{+}=Q_{\omega}(1,0)$. In the case where measurements are performed at $m_{f}$ different frequencies, Eq. generalizes to

$$
\rho_{I}^{\left(\left\{M_{i}\right\},\left\{\alpha_{i}^{-}\right\},\left\{\omega_{i}\right\}\right)}(x)=\rho_{I}(x) \prod_{i=1}^{m_{f}} \frac{W_{\omega_{i}}\left(M_{i}, \alpha_{i}^{-} ; x\right)}{Q_{\omega_{i}}\left(M_{i}, \alpha_{i}^{-}\right)} .
$$


The probability density $\rho_{I}^{\left(\left\{M_{i}\right\},\left\{\alpha_{i}^{-}\right\},\left\{\omega_{i}\right\}\right)}(x)$ after $M$ measurements performed at $m_{f}$ different driving frequencies depends on the frequencies $\left\{\omega_{i}\right\}=\left\{\omega_{1}, \ldots, \omega_{m_{f}}\right\}$, the number of measurements at each frequency $\left\{M_{i}\right\}=$ $\left\{M_{1}, \ldots, M_{m_{f}}\right\}$, and the number of times $|-\rangle$ was measured at each frequency $\left\{\alpha_{i}^{-}\right\}=\left\{\alpha_{1}^{-}, \ldots, \alpha_{m_{f}}^{-}\right\}$. Eq. (33) gives the distribution of nuclear spin eigenvalues for any sequence of $M$ measurements, i.e., without randomization of the nuclear-spin system in between measurements.

\section{B. Measurement schemes}

In this subsection we describe different measurement schemes. One main characteristic of the schemes is whether we have unconditional evolution of the nuclearspin density matrix between measurements (one waits for the nuclear-spin system to rerandomize between subsequent measurements), or whether we have conditional evolution, i.e., the nuclear-spin system is assumed to be static between measurements.

\section{Unconditional scheme}

The simplest scheme is to measure only once at one single driving frequency $\omega$. If the outcome is $|-\rangle$, the nuclear-spin distribution after the measurement is given by Eq. (26); the FWHM $\left(2 \sigma_{0} \sqrt{2 \ln 2} \approx 2 \sigma_{0}\right)$ of the initial distribution will have been narrowed by a factor $\approx j / 4 \sigma_{0}$ (the nuclear-spin distribution will approximately be a Lorentzian with FWHM of $j / 2$ ). For $j \ll \sigma_{0}$ and $\omega=2 x_{0}$, the probability $P_{\omega}^{-}$to measure $|-\rangle$in the first measurement is $P_{\omega=2 x_{0}}^{-} \approx j / 6 \sigma_{0}$ (the exact formula is given in Eq.(28)). If the measurement outcome is $|-\rangle$, we stop measuring. Otherwise, we wait for the system to rerandomize (in contrast to the conditional schemes) and perform another measurement. This is repeated until $|-\rangle$ is measured for the first time. On average one needs to perform $M^{\prime} \approx 6 \sigma_{0} / j$ measurements in order to narrow by a factor of $\approx j / 4 \sigma_{0}$ (we write $M^{\prime}$ because this number of measurements should not be confused with the number of measurements $M$ used above in the case of measurements performed without rerandomization in between). If the driving frequency $\omega$ is far from the center $x_{0}$ of the initial Gaussian distribution, the number of required measurements increases by a factor of $\exp \left(\left(x_{0}-\omega / 2\right)^{2} / 2 \sigma_{0}^{2}\right)$. This always leads to a narrowed distribution which is centered around $\omega / 2$. Thus, with this scheme it is possible to choose the center of the nuclear-spin distribution after the measurement. This unconditional measurement scheme is the one which should be easiest to implement in an experiment since one only needs to measure once at one single frequency. However, if measurements at several different frequencies can be performed, a systematic narrowing of the distribution can be implemented as we show next.

\section{Adaptive conditional scheme}

The probability of measuring $|-\rangle$ in a measurement is determined by the overlap of the Lorentzian $L_{\omega}(x)$ and the probability density of eigenvalues $\rho_{I}^{\left(M, \alpha^{-}, \omega\right)}(x)$ (for the first measurement this probability is $P_{\omega}^{-}$, which is given in Eq.(28)). Then, if we have the outcome $|-\rangle$ for a measurement at driving frequency $\omega, \rho_{I}^{\left(M, \alpha^{-}, \omega\right)}(x)$ as a function of $x$ becomes peaked around $\omega / 2$ ( since $L_{\omega}(x)$ is centered around $\left.x=\omega / 2\right)$, the overlap of the Lorentzian $L_{\omega}(x)$ and $\rho_{I}^{\left(M, \alpha^{-}, \omega\right)}(x)$ increases and therefore the probability to measure $|-\rangle$ in a subsequent measurement also grows. If, on the other hand, we have outcome $|+\rangle$, the term $1-L_{\omega}(x)$ causes a dip in $\rho_{I}^{\left(M, \alpha^{-}, \omega\right)}(x)$ at $x=\omega / 2$, the overlap of the Lorentzian $L_{\omega}(x)$ and $\rho_{I}^{\left(M, \alpha^{-}, \omega\right)}(x)$ decreases and thus the probability to measure $|-\rangle$ in a subsequent measurement with the same driving frequency $\omega$ also decreases. Since it is the measurement outcome $|-\rangle$ that primarily leads to narrowing, the measurement scheme should maximize the probability to measure $|-\rangle$. This can be achieved by changing the driving frequency $\omega$ always in such a way that before each measurement $L_{\omega}(x)$ and the nuclear-spin distribution $\rho_{I}^{\left(M, \alpha^{-}, \omega\right)}(x)$ have their maximum at the same $x$, i.e., set $\omega / 2=x_{\max }$, where $x_{\max }$ is the $x$ for which $\rho_{I}^{\left(M, \alpha^{-}, \omega\right)}(x)$ has a maximum. Thanks to the adaptive driving frequency $\omega$, the probability $P_{\omega}^{-}$to measure $|-\rangle$ is $\approx j / 6 \sigma_{0}$ in each measurement until $|-\rangle$ is measured for the first time. Without adapting, i.e., when measuring always at the same driving frequency $\omega, P_{\omega}^{-}$decreases, as explained above (as long as we do not measure $|-\rangle$ ). After measuring $|-\rangle$ for the first time, the probability $P_{\omega}^{-}$ to measure $|-\rangle$ increases. Every time the measurement outcome is $|-\rangle$, the distribution $\rho_{I}^{\left(M, \alpha^{-}, \omega\right)}(x)$ is multiplied by $L_{\omega}(x)$ and becomes narrower (since $L_{\omega}(x)^{\alpha^{-}}$ has a FWHM of $\left.(j / 2) \sqrt{2^{1 / \alpha^{-}}-1}\right)$. However, the measurement outcome $|+\rangle$, for which $\rho_{I}^{\left(M, \alpha^{-}, \omega\right)}(x)$ is multiplied by $1-L_{\omega}(x)$, is still more likely and leads to a small widening of the distribution. Our simulations of this measurement scheme do, however, show that after $|-\rangle$ has been measured several times, the nuclear spin distribution is narrowed by more than a factor $j / 4 \sigma_{0}$.

This adaptive scheme was first proposed in an optical setup by Stepanenko et al. in Ref. 30. This scheme requires that $x_{\max }$ can be calculated (or read from a table) between subsequent measurements and that the driving frequency $\omega$ can be tuned with a precision that is better than the width of the nuclear-spin distribution before each measurement. For this adaptive scheme (and other conditional schemes) to work, it is important that the nuclear-spin system does not randomize during the course of the complete measurement, i.e., the complete measurement must be carried out within a time that is shorter than the time scale for nuclear spin dynamics. We thus assume that the nuclear-spin system (viz. $\delta h^{z}$ ) has 
no internal dynamics between the single measurements of $\tau^{z}(t)$, but only changes due to the measurements performed on the two-electron system, i.e., due to single measurements of $\tau^{z}(t)$. We expect $\delta h^{z}$ to vary on the time scale of nuclear spin diffusion out of the dot, which is on the order of seconds for nuclear spins surrounding donor impurities in GaAs $\stackrel{27}{\underline{27}}$ However, there may be other

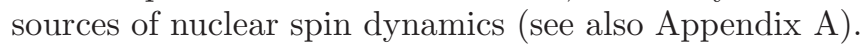
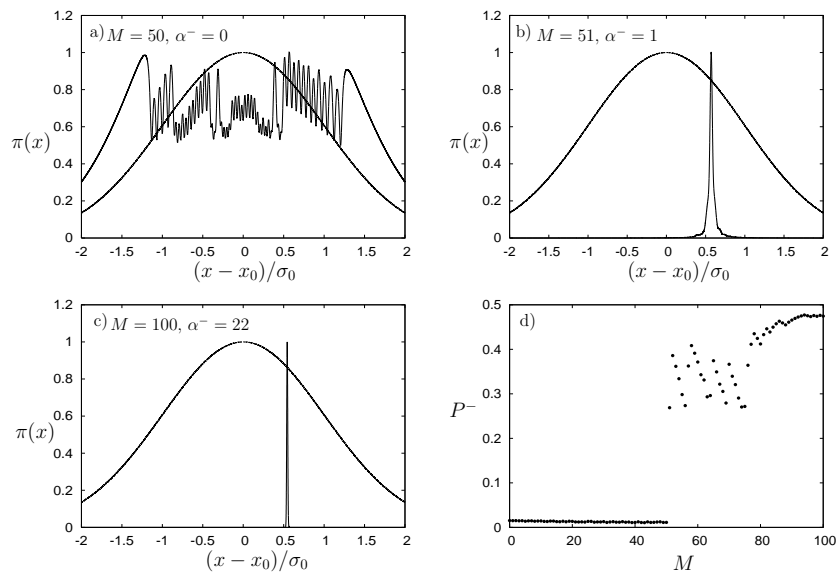

FIG. 2: In this figure we show a typical ${ }^{39}$ sequence of the rescaled probability density of eigenvalues $\pi(x)=\rho_{I}^{\left(\left\{M_{i}\right\},\left\{\alpha_{i}^{-}\right\},\left\{\omega_{i}\right\}\right)}(x) / \max \left(\rho_{I}^{\left(\left\{M_{i}\right\},\left\{\alpha_{i}^{-}\right\},\left\{\omega_{i}\right\}\right)}(x)\right)$ for the adaptive conditional scheme. Here, $\rho_{I}^{\left(\left\{M_{i}\right\},\left\{\alpha_{i}^{-}\right\},\left\{\omega_{i}\right\}\right)}(x)$ is given in Eq. (33). We have $x=\delta h_{n}^{z}+\delta b^{z}, j / \sigma_{0}=1 / 10$ and in a) - c) the initial Gaussian distribution (with FWHM $2 \sigma_{0} \sqrt{2 \ln 2} \approx 2 \sigma_{0}$ ) is plotted for reference. a) Up to $M=50$ measurements the outcome is never $|-\rangle$ and thus each measurement "burns a hole" into the distribution where it previously had its maximum. b) In the $51^{\text {st }}$ measurement the outcome is $|-\rangle$ which leads to a narrowed distribution of nuclear spin eigenvalues (peak centered at $\approx 0.5$ ) with a FWHM that is reduced by a factor $\approx j / 4 \sigma_{0}$. c) Adapting the driving frequency $\omega$ to this peak, i.e., setting $\omega / 2=x_{\max }$ in subsequent measurements, leads to further narrowing every time $|-\rangle$ is measured. In this example the final FWHM is $\approx \sigma_{0} / 100$, i.e., the distribution has been narrowed by a factor $\approx j / 10 \sigma_{0}$. d) The probability $P^{-}$to measure $|-\rangle$jumps up after the $51^{\text {st }}$ measurement and after $|-\rangle$ is measured several more times, this probability saturates close to $1 / 2$.

In figure 2 we show a typica ${ }^{39}$ sequence of nuclear spin distributions for the adaptive scheme with total number of measurements $M=100$ and $j / \sigma_{0}=1 / 10$. We see ( figure 2 (a)) that up to $M=50$ the measurement outcome is never $|-\rangle$ and thus each measurement "burns a hole" into the distribution where it previously had its maximum. In the $51^{\text {st }}$ measurement (figure2(b)) the outcome is $|-\rangle$, which narrows the distribution by a factor of $\approx j / 4 \sigma_{0}$. Adapting the driving frequency $\omega$ to this peak, i.e., setting $\omega / 2=x_{\max }$ in subsequent measurements, leads to further narrowing, i.e., to a total narrowing by more than a factor $j / 4 \sigma_{0}$ (figure 2(c)). In this example we have $\alpha^{-}=22$ after $M=100$ measurements and the final FWHM is $\approx \sigma_{0} / 100$, i.e., the distribution has been narrowed by a factor $\approx j / 10 \sigma_{0}$. In figure $2(\mathrm{~d})$ the probability $P^{-}$to measure $|-\rangle$before each measurement is shown. After the first time $|-\rangle$ is measured, $P^{-}$jumps up and after several more times $|-\rangle$ was measured, it saturates close to $1 / 2 . P^{-}$is a good signature of the distribution's width. As the width of the distribution goes to zero, $P^{-}$approaches $1 / 2$. This adaptive conditional scheme is more intricate than the unconditional scheme, but allows one to narrow by more than a factor $j / 4 \sigma_{0}$.

\section{Other conditional schemes}

Other possible measurement schemes involve measurements at several frequencies, as in the adaptive scheme. One may either choose a fixed number of frequencies within one or two $\sigma_{0}$ and measure several times at each frequency (without randomization between the measurements) or sweep the frequency, i.e., measure only once at each frequency but vary the frequency only in small steps. Based on numerical simulations of these schemes, we find that the typical number of measurements to narrow by a factor of $j / \sigma_{0}$ is greater than in the adaptive or the unconditional (single-frequency) schemes.

\section{Time-domain measurement scheme}

We note that when a complete measurement of one of the correlators discussed in Sec. IV] is performed with perfect resolution in time and perfect accuracy, this would also determine the state of the nuclear spin system and thus narrow the distribution of nuclear spin states. This is because the frequency of the oscillating correlators is given by $\sqrt{J^{2}+4\left(\delta h_{n}^{z}\right)^{2}}$ and thus measuring the frequency of the correlator determines the eigenvalue $\delta h_{n}^{z}$ of the nuclear-spin system. However, it may be possible to perform a weak measurement of the decay of the correlators and thus also to see the prolongation of the decay after applying a narrowing scheme. To understand in detail the effect of measurements in the time domain, further study is required. Narrowing through measurement of the correlators is a time-domain measurement. In contrast, the narrowing schemes we have proposed above are frequency-domain measurements. If the frequency resolution is better than the time resolution, our method would most likely be more suitable.

\section{CORRELATION FUNCTIONS IN THE $S^{z}=0$ SUBSPACE}

In this section we investigate the Hamiltonian $H_{0}$ of Eq. (11) with static exchange coupling $J$. Using this Hamiltonian we wish to calculate correlation functions for several observables in the subspace of zero total spin in the $z$-direction. In our previous work ${ }^{18}$ we calculated 
the time evolution of a particular correlator involving the states $|S\rangle$ and $\left|T_{0}\right\rangle$. However, there are four additional independent correlators involving the $x$ and $y$ components of pseudo-spin which require a separate calculation. Quite surprisingly, it will turn out that these correlators have different decay behavior in time. The correlators we calculate here show the decoherence properties of the pseudo-spin states under the influence of the hyperfine interaction. There may be additional sources of decoherence which we do not consider here, such as orbital dephasing, corrections to the effective Hamiltonian, 18 the coupling of the QPC to the dot spins $\stackrel{40}{=}$ etc. The results of this section will help to give requirements on the parameters of the system in order to initialize in the state $|\uparrow \downarrow\rangle$ and to assess the fidelity of a $\sqrt{\text { SWAP }}$ operation with static $J$ (see Sec. V).

Diagonalizing $H_{0}$ gives the following eigenvalues and eigenvectors

$$
\begin{aligned}
E_{n}^{ \pm} & =\frac{J}{2} \pm \frac{1}{2} \sqrt{J^{2}+\Omega_{n}^{2}} \\
\left|E_{n}^{ \pm}\right\rangle & =\frac{\left(\Omega_{n} / 2\right)|S\rangle+E_{n}^{ \pm}\left|T_{0}\right\rangle}{\sqrt{\left(E_{n}^{ \pm}\right)^{2}+\left(\Omega_{n} / 2\right)^{2}}} \otimes|n\rangle,
\end{aligned}
$$

where again $|n\rangle$ is an eigenstate of the operator $\delta h^{z}$ with $\delta h^{z}|n\rangle=\delta h_{n}^{z}|n\rangle$. At $t=0$ we fix the electron system in an arbitrary superposition of $\left|T_{0}\right\rangle$ and $|S\rangle$

$$
\left|\psi_{e}(t=0)\right\rangle=|A\rangle ;|A\rangle=\cos \frac{\theta_{A}}{2}|S\rangle+e^{i \varphi_{A}} \sin \frac{\theta_{A}}{2}\left|T_{0}\right\rangle .
$$

The nuclear-spin system is again in a general state (see Sec. IA . As will be shown in Sec. D it is possible, in principle, to initialize to an arbitrary state in the subspace spanned by $\left|T_{0}\right\rangle$ and $|S\rangle$. The probability to find the electron spins in a state $|B\rangle$ at $t>0$ is given by the correlation function:

$$
C_{B A}(t)=\sum_{n} \rho_{I}(n)\left|\left\langle n\left|\otimes\left\langle B\left|e^{-i H_{0} t}\right| A\right\rangle \otimes\right| n\right\rangle\right|^{2},
$$

where $\rho_{I}(n)=\sum_{i} p_{i}\left|a_{n}^{i}\right|^{2}$. The correlation function has the following symmetry: $C_{B A}(t)=C_{A B}(-t)$, and if $|B\rangle$ and $|D\rangle$ are orthogonal states we have $C_{B A}(t)=1-$ $C_{D A}(t)$. Further, we may decompose $C_{B A}(t)$ into the sum of a time-independent term $\overline{C_{B A}^{n}}$ and an interference term $C_{B A}^{\text {int }}(t)$ :

$$
C_{B A}(t)=\overline{C_{B A}^{n}}+C_{B A}^{\mathrm{int}}(t),
$$

where the overbar is defined in Eq. (10).

We have further $C_{B A}^{n}=C_{B A}\left(\delta h_{n}^{z}\right)=C_{B A}(x)$. Performing the continuum limit as described in Eq. (11) we obtain for the correlation function

$$
\begin{aligned}
C_{B A}(t) & =\int_{-\infty}^{\infty} d x \rho_{I ; \sigma_{0}, x_{0}}(x)\left(C_{B A}(x)+C_{B A}^{\mathrm{int}}(x, t)\right) \\
& =C_{B A}^{\infty}+C_{B A}^{\mathrm{int}}(t) .
\end{aligned}
$$

Here, $C_{B A}^{\infty}$ is the assymptotic value of the correlator $C_{B A}(t)$ for $t \rightarrow \infty$.

We have calculated correlation functions for the following states: $|S\rangle \rightarrow\left|\tau^{z}=-1\right\rangle,\left|T_{0}\right\rangle \rightarrow\left|\tau^{z}=+1\right\rangle$, $|X\rangle \rightarrow\left|\tau^{x}=+1\right\rangle=\frac{1}{\sqrt{2}}\left(\left|T_{0}\right\rangle+|S\rangle\right),|Y\rangle \rightarrow\left|\tau^{y}=+1\right\rangle=$ $\frac{1}{\sqrt{2}}\left(\left|T_{0}\right\rangle+i|S\rangle\right)$. The frequency in the interference term is always given by $s(x)=\sqrt{J^{2}+4 x^{2}}$. In Table प we list the integrands according to the notation in Eq. (39). From the Heisenberg equation of motion we find $\frac{d \tau^{x}}{d t}=-J \tau^{y}$, which leads to relations for the correlators. In the notation used in Table \we obtain $\frac{d C_{X X}}{d t}=$ $-J\left(C_{Y X}-\frac{1}{2}\right)$, which is satisfied by the results shown in Table \ Similar relations can be derived for the other correlators and used to check the results in Table 【 We

\begin{tabular}{|c||c|c|}
\hline$C_{B A}(t)$ & $C_{B A}(x)$ & $C_{B A}^{\mathrm{int}}(x, t)$ \\
\hline \hline$C_{T_{0} S}(t)$ & $\frac{2 x^{2}}{s(x)^{2}}$ & $-\frac{2 x^{2}}{s(x)^{2}} \cos (s(x) t)$ \\
\hline$C_{T_{0} X}(t)$ & $\frac{1}{2}+\frac{J x}{s(x)^{2}}$ & $-\frac{J x}{s(x)^{2}} \cos (s(x) t)$ \\
\hline$C_{T_{0} Y}(t)$ & $\frac{1}{2}$ & $\frac{x}{s(x)} \sin (s(x) t)$ \\
\hline$C_{Y X}(t)$ & $\frac{1}{2}$ & $\frac{J}{2 s(x)} \sin (s(x) t)$ \\
\hline$C_{Y Y}(t)$ & $\frac{1}{2}$ & $\frac{1}{2} \cos (s(x) t)$ \\
\hline$C_{X X}(t)$ & $\frac{1}{2}+\frac{2 x^{2}}{s(x)^{2}}$ & $\frac{J^{2}}{2 s(x)^{2}} \cos (s(x) t)$ \\
\hline
\end{tabular}

TABLE I: Functions $C_{B A}(x)$ and $C_{B A}^{\text {int }}(x, t)$ according to the notation of Eq. (39) for different correlators (with $s(x)=$ $\left.\sqrt{J^{2}+4 x^{2}}\right)$. $C_{X X}(t)$ is a linear combination of other correlators.

see that $C_{X X}(t)$ is a linear combination of other correlators: $C_{X X}(t)=C_{Y Y}(t)+C_{T_{0} S}(t)$. For $C_{T_{0} X}$ and $C_{T_{0} Y}$ the interference term is an odd function in $x$. Thus, the time dependence vanishes for $x_{0}=0$ and we have $C_{T_{0} X}=C_{T_{0} Y}=1 / 2$ for all $t$. In general, the integral in Eq. (39) is difficult to solve exactly. Thus, we concentrate on several interesting limits. We illustrate this for the case of $C_{Y X}(t)$ and give results for the other correlators. We have

$$
\begin{aligned}
C_{Y X}(t) & =\frac{1}{2}+\operatorname{Im}\left[\tilde{C}_{Y X}^{\mathrm{int}}\right] \\
\tilde{C}_{Y X}^{\mathrm{int}} & =\int_{-\infty}^{\infty} \rho_{I ; \sigma_{0}, x_{0}}(x) \frac{J}{2 s(x)} e^{i s(x) t} .
\end{aligned}
$$

In the regime of $\left|x_{0}\right| \gg \sigma_{0}$ the main contribution to the integral comes from a narrow region around $x_{0}$ and we may approximate $\frac{J}{2 s(x)} \approx \frac{J}{2 \omega_{0}}$ where $\omega_{0}=s\left(x_{0}\right)$ and in the frequency term $s(x) \approx \omega_{0}+\frac{4 x_{0}}{\omega_{0}}\left(x-x_{0}\right)+\ldots$. For this to be a good approximation, we require $\frac{2 J^{2}}{w_{0}^{3}}(x-$ $\left.x_{0}\right)^{2} t \ll 1$. We use $\left(x-x_{0}\right)^{2} \approx \sigma_{0}^{2}$ and thus obtain for 
the correlator and the range of validity in this limit

$$
\begin{aligned}
C_{Y X}^{\mathrm{int}}(t)= & \frac{J}{2 \omega_{0}} e^{-\frac{1}{2}\left(\frac{t}{t_{0}^{\prime \prime}}\right)^{2}} \sin \left(\omega_{0} t\right), \\
& t_{0}^{\prime \prime}=\frac{\omega_{0}}{4\left|x_{0}\right| \sigma_{0}}, \omega_{0}=\sqrt{J^{2}+4 x_{0}^{2}}, \\
& \left|x_{0}\right| \gg \sigma_{0}, \quad t \ll \frac{\left(J^{2}+4 x_{0}^{2}\right)^{3 / 2}}{2 J^{2} \sigma_{0}^{2}} .
\end{aligned}
$$

The results for the other correlators are (with the same range of validity)

$$
\begin{aligned}
& C_{T_{0} S}^{\mathrm{int}}(t)=-\frac{2 x_{0}^{2}}{\omega_{0}^{2}} e^{-\frac{1}{2}\left(\frac{t}{t_{0}^{\prime \prime}}\right)^{2}} \cos \left(\omega_{0} t\right), \\
& C_{T_{0} X}^{\mathrm{int}}(t)=-\frac{J x_{0}}{\omega_{0}^{2}} e^{-\frac{1}{2}\left(\frac{t}{t_{0}^{\prime \prime}}\right)^{2}} \cos \left(\omega_{0} t\right), \\
& C_{T_{0} Y}^{\mathrm{int}}(t)=\frac{x_{0}}{\omega_{0}} e^{-\frac{1}{2}\left(\frac{t}{t_{0}^{\prime \prime}}\right)^{2}} \sin \left(\omega_{0} t\right), \\
& C_{Y Y}^{\mathrm{int}}(t)=\frac{1}{2} e^{-\frac{1}{2}\left(\frac{t}{t_{0}^{\prime \prime}}\right)^{2}} \cos \left(\omega_{0} t\right) .
\end{aligned}
$$

In this limit we obtain a Gaussian decay for all correlators on a time scale $t_{0}^{\prime \prime}=\frac{\omega_{0}}{4\left|x_{0}\right| \sigma_{0}}$ which grows with the absolute value of the exchange coupling $|J|$ and with $1 / \sigma_{0}$. The long-time saturation value is $1 / 2$ for $C_{Y X}$. For some of the other correlators we find non-trivial parameterdependent saturation values. In the limit of $\left|x_{0}\right| \gg \sigma_{0}$ we obtain these correlators by the same approximation as for the interference term, i.e. we set $C_{B A}(x)=C_{B A}\left(x_{0}\right)$ and obtain

$$
\begin{aligned}
C_{T_{0} S}^{\infty} & =\frac{2 x_{0}^{2}}{J^{2}+4 x_{0}^{2}} ; \quad\left|x_{0}\right| \gg \sigma_{0}, \\
C_{T_{0} X}^{\infty} & =\frac{1}{2}+\frac{J x_{0}}{J^{2}+4 x_{0}^{2}} ; \quad\left|x_{0}\right| \gg \sigma_{0}, \\
C_{T_{0} Y}^{\infty} & =C_{Y X}^{\infty}=C_{Y Y}^{\infty}=\frac{1}{2} .
\end{aligned}
$$

For large $J$ the saturation value is quadratic in $x_{0} / J$ for $C_{T_{0} S}$ and linear for $C_{T_{0} X}$. The saturation value for $C_{T_{0} S}$ goes to zero for $|J| \gg\left|x_{0}\right|$ and for $C_{T_{0} X}$ approaches $1 / 2 . C_{T_{0} X}^{\infty}$ reaches extrema equal to $\frac{1}{2}+\frac{1}{4} \operatorname{sign}\left(J x_{0}\right)$ for $|J|=2\left|x_{0}\right|$.

Next we consider Eq. (39) for $|J| \gg \max \left(\left|x_{0}\right|, \sigma_{0}\right)$ and find

$$
\begin{gathered}
s(x)=\sqrt{J^{2}+4 x^{2}} \approx|J|+\frac{2 x^{2}}{|J|}, \\
\frac{J}{2 s(x)}=\frac{J}{2 \sqrt{J^{2}+4 x^{2}}} \approx \operatorname{sign}(J)\left(\frac{1}{2}-\frac{x^{2}}{J^{2}}\right) .
\end{gathered}
$$

For Eq. (53) we have the additional requirement that $t \ll \frac{|J|^{3}}{2 \max \left(x_{0}^{4}, \sigma_{0}^{4}\right)}$. Under these approximations we find the following result:

$$
\begin{gathered}
\tilde{C}_{Y X}^{\mathrm{int}}(t)=\operatorname{sign}(J)\left(\frac{1}{2} \xi(t)-\frac{\sigma_{0}^{2}}{J^{2}} \xi^{3}(t)-\frac{x_{0}^{2}}{J^{2}} \xi^{5}(t)\right) \exp \left(i|J| t-\frac{x_{0}^{2}}{2 \sigma_{0}^{2}}\left(1-\xi^{2}(t)\right)\right), \\
\xi(t)=\left(1-i \frac{t}{t_{0}^{\prime}}\right)^{-1 / 2}, t_{0}^{\prime}=\frac{|J|}{4 \sigma_{0}^{2}}, \quad|J| \gg \max \left(\left|x_{0}\right|, \sigma_{0}\right), \quad t \ll \frac{|J|^{3}}{2 \max \left(x_{0}^{4}, \sigma_{0}^{4}\right)}
\end{gathered}
$$

At short times we expand $\xi^{2}(t) \sim 1+i \frac{t}{t_{0}^{\prime}}-\left(\frac{t}{t_{0}^{\prime}}\right)^{2}$. Keeping only lowest order in $t / t_{0}^{\prime}$ in the prefactor and second order in the frequency term we obtain

$$
\begin{aligned}
& C_{Y X}^{\operatorname{int}}(t)= \operatorname{sign}(J) \frac{1}{2} e^{-\frac{1}{2}\left(\frac{t}{t_{0}^{\prime \prime}}\right)^{2}} \sin \left(\omega_{0}^{\prime} t\right), \\
& t_{0}^{\prime \prime} \approx \frac{|J|}{4\left|x_{0}\right| \sigma_{0}}, \omega_{0}^{\prime}=|J|+\frac{2\left(x_{0}^{2}+\sigma_{0}^{2}\right)}{|J|}, \\
& t \ll t_{0}^{\prime}=\frac{|J|}{4 \sigma_{0}^{2}}, \quad|J| \gg \max \left(\left|x_{0}\right|, \sigma_{0}\right) .
\end{aligned}
$$

The $\left|x_{0}\right| \gg \sigma_{0}$ limit of this result agrees with the $|J| \gg\left|x_{0}\right|$ limit of Eq. [43). Again, we have a Gaussian decay on the same time scale $t_{0}^{\prime \prime}$ as in Eq. (43) $\left(\omega_{0}=\sqrt{J^{2}+4 x_{0}^{2}} \sim|J|\right.$ for $\left.|J| \gg\left|x_{0}\right|\right)$. One interesting feature of this correlator is the fact that there is a change of phase by $\pi$ when the sign of the exchange coupling $J$ changes. This feature offers the possibility of measuring $J$ even for small values of $J$ through a measurement of this correlator. We also list the other correlators in this regime:

$$
\begin{aligned}
& C_{T_{0} S}^{\mathrm{int}}(t)=-\frac{2\left(x_{0}^{2}+\sigma_{0}^{2}\right)}{J^{2}} e^{-\frac{1}{2}\left(\frac{t}{t_{0}^{\prime \prime}}\right)^{2}} \cos \left(\omega_{0}^{\prime} t\right), \\
& C_{T_{0} X}^{\mathrm{int}}(t)=-\frac{x_{0}}{J} e^{-\frac{1}{2}\left(\frac{t}{t_{0}^{\prime \prime}}\right)^{2}} \cos \left(\omega_{0}^{\prime} t\right), \\
& C_{T_{0} Y}^{\mathrm{int}}(t)=\frac{x_{0}}{|J|} e^{-\frac{1}{2}\left(\frac{t}{t_{0}^{\prime \prime}}\right)^{2}} \sin \left(\omega_{0}^{\prime} t\right), \\
& C_{Y Y}^{\mathrm{int}}(t)=\frac{1}{2} e^{-\frac{1}{2}\left(\frac{t}{t_{0}^{\prime \prime}}\right)^{2}} \cos \left(\omega_{0}^{\prime} t\right) .
\end{aligned}
$$


Finally, we are also interested in the behavior for large t. Thus, we expand Eq. (55) for large times $\xi\left(t \gg t_{0}^{\prime}\right) \sim$ $e^{i \pi / 4} \sqrt{t_{0}^{\prime} / t}$ and obtain

$$
\begin{aligned}
C_{Y X}^{\operatorname{int}}(t) \sim & \operatorname{sign}(J) e^{-\frac{x_{0}^{2}}{2 \sigma_{0}^{2}}} \frac{\sqrt{|J|} \sin \left(|J| t+\frac{\pi}{4}\right)}{4 \sigma_{0} t^{\frac{1}{2}}}, \\
& t \gg t_{0}^{\prime}=\frac{|J|}{4 \sigma_{0}^{2}},|J| \gg \max \left(\left|x_{0}\right|, \sigma_{0}\right) .
\end{aligned}
$$

For the other correlators we find

$$
\begin{aligned}
& C_{T_{0} S}^{\text {int }}(t) \sim-e^{-\frac{x_{0}^{2}}{2 \sigma_{0}^{2}}} \frac{\cos \left(|J| t+\frac{3 \pi}{4}\right)}{4 \sigma_{0} \sqrt{|J|} t^{\frac{3}{2}}}, \\
& C_{T_{0} X}^{\text {int }}(t) \sim-\operatorname{sign}(J) e^{-\frac{x_{0}^{2}}{2 \sigma_{0}^{2}}} \frac{x_{0} \sqrt{|J|} \cos \left(|J| t+\frac{3 \pi}{4}\right)}{8 \sigma_{0}^{3} t^{\frac{3}{2}}}(67) \\
& C_{T_{0} Y}^{\mathrm{int}}(t) \sim e^{-\frac{x_{0}^{2}}{2 \sigma_{0}^{2}}} \frac{x_{0} \sqrt{|J|} \sin \left(|J| t+\frac{3 \pi}{4}\right)}{8 \sigma_{0}^{3} t^{\frac{3}{2}}}, \\
& C_{Y Y}^{\mathrm{int}}(t) \sim e^{-\frac{x_{0}^{2}}{2 \sigma_{0}^{2}}} \frac{\sqrt{|J|} \cos \left(|J| t+\frac{\pi}{4}\right)}{4 \sigma_{0} t^{\frac{1}{2}}} .
\end{aligned}
$$

Thus, the transverse components of the pseudo-spin have a slower decay $\left(\sim t^{-1 / 2}\right)$ than the longitudinal component $\left(\sim t^{-3 / 2}\right)$. This results from the fact that the Hamiltonian only has fluctuations along only one direction.

\section{ANALYSIS OF $\sqrt{\text { SWAP }}$}

In this section we analyze the $\sqrt{\text { SWAP }}$ gate using the correlation functions derived in the previous section, i.e., we analyze the $\sqrt{\text { SWAP }}$ gate taking into account the hyperfine-induced decoherence. The $\sqrt{\text { SWAP }}$ gate and single-qubit operations can be used to perform the quantum XOR gate (CNOT) which, in combination with single-qubit operations, is sufficient for universal quantum computation ${ }^{1.41}$ In Ref. 9 implementation of $\sqrt{\text { SWAP }}$ has been demonstrated. However, in these experiments there was a contrast reduction of $\sim 40 \%$. Here we show that taking into account hyperfine induced decoherence, still near-unit fidelity can be obtained for this operation.

The Hamiltonian of Eq. (10) induces unitary time evolution on the states of the system: $|\psi(t)\rangle=U(t)|\psi(0)\rangle$ with $U(t)=T \exp \left(-i \int_{0}^{t} H\left(t^{\prime}\right) d t^{\prime}\right)$. We assume that $J$ and $x_{0}$ can be switched adiabatically ${ }^{42}$ on a time scale that is much shorter than the time required for the gate operation and thus the time evolution operator at time $\tau_{s}$ has the form

$$
U_{s}=\exp \left(-i \tau_{s} H\right)
$$

In a Bloch-sphere picture this operator induces a rotation about an axis in the plane spanned by eigenstates of $\tau^{x}$ and $\tau^{z},|X\rangle=|\uparrow \downarrow\rangle$ and $|S\rangle=(|\uparrow \downarrow\rangle-|\downarrow \uparrow\rangle) / \sqrt{2} \underline{31}$
The axis of rotation is determined by the parameters $J$ and $x_{0}$. Through such an operation any state may be rotated into any other state on the Bloch sphere. Thus, it is possible to rotate from $|S\rangle$ to any initial state in the subspace of $S^{z}=0$ by a single operation. This is important since initialization to the singlet is feasible by preparing a ground-state singlet with both electrons on the same dot and then changing the bias $\frac{9}{\underline{9}}$ We now investigate initialization to the state $|X\rangle$ taking into account hyperfine-induced decoherence. The scheme we propose here is different from the one used in Ref. 9, where adiabatic passage from the singlet to the $|\uparrow \downarrow\rangle$-state is used. Our scheme requires control of $x_{0}$. We assume the system to be in the singlet state $|S\rangle$ at $t=0$ and then switch $J$ and $x_{0}$ such that $J=-2 x_{0}$ and $\left|x_{0}\right| \gg \sigma_{0}$. In a Blochsphere picture, this corresponds to a rotation about an axis that halves the angle between $|S\rangle$ and $|X\rangle$. Since $C_{X S}(t)=C_{S X}(-t)=1-C_{T_{0} X}(-t)$ we have, for the above choice of parameters, according to Eqs. 477 and (51):

$$
\begin{aligned}
& C_{X S}(t)= \frac{1}{2}+\frac{1}{4}\left(1-\cos (\sqrt{2}|J| t) e^{-\frac{1}{2}\left(\frac{t}{t_{0}^{\prime \prime}}\right)^{2}}\right), \\
& J=-2 x_{0}, \quad\left|x_{0}\right| \gg \sigma_{0}, \\
& t_{0}^{\prime \prime}=\frac{1}{\sqrt{2} \sigma_{0}}, \quad t \ll \frac{\left(J^{2}+4 x_{0}^{2}\right)^{3 / 2}}{2 J^{2} x_{0}^{2}} .
\end{aligned}
$$

This correlator reaches its maximum for $\sqrt{2}|J| t=\pi$, i.e., at $\tau_{s}=\frac{\pi}{\sqrt{2}|J|}$. The time scale for the Gaussian decay is $t^{\prime \prime}=\frac{1}{\sqrt{2} \sigma_{0}}$. To approach unit fidelity we therefore require $|J| \gg \sigma_{0}$, which is the case in the range of validity of the above correlator since $\left|x_{0}\right| \gg \sigma_{0}$ and $J$ and $x_{0}$ are of the same order. At $t=\tau_{s}$ we switch $J$ to zero and since $|X\rangle \otimes|n\rangle$ is an eigenstate of the remaining Hamiltonian, the system remains in this product state, untouched by decoherence induced via the nuclear spins. This scheme thus provides a way to initialize the double quantum dot system to the state $|X\rangle=\frac{1}{\sqrt{2}}\left(\left|T_{0}\right\rangle+|S\rangle\right)=|\uparrow \downarrow\rangle$, where arrows denote the z-component of the electron spin in each dot. In the same way, it is also possible to initialize in the state $|-X\rangle=\left|\tau^{x}=-1\right\rangle=\frac{1}{\sqrt{2}}\left(\left|T_{0}\right\rangle-|S\rangle\right)=|\downarrow \uparrow\rangle$ by switching to $J=2 x_{0}$.

It was already proposed in Ref. 1 to implement the $\sqrt{\text { SWAP }}$ gate by pulsing the exchange interaction $J$ between the two dots. Here we give a detailed analysis of the $\sqrt{\text { SWAP }}$ gate taking into account hyperfine-induced decoherence.

The SWAP operation acts on the basis of the twoelectron system as: $|\downarrow \downarrow\rangle \rightarrow|\downarrow \downarrow\rangle,|\downarrow \uparrow\rangle \rightarrow|\uparrow \downarrow\rangle,|\uparrow \downarrow\rangle \rightarrow$ $|\downarrow \uparrow\rangle,|\uparrow \uparrow\rangle \rightarrow|\uparrow \uparrow\rangle$. The SWAP is an operation that acts only on the subspace of $S^{z}=0$ and leaves the states $|\uparrow \uparrow\rangle$ and $|\downarrow \downarrow\rangle$ unchanged. In the system we consider this is naturally implemented through the large Zeeman splitting that separates $|\uparrow \uparrow\rangle$ and $|\downarrow \downarrow\rangle$ from the singlet and the $S^{z}=0$ triplet. In order to analyze the SWAP in the $S^{z}=0$ subspace we consider the regime 
of $|J| \gg \max \left(x_{0}, \sigma_{0}\right)$. The correlator $C_{-X, X}(t)$ gives the probability of being in the state $|-X\rangle=|\downarrow \uparrow\rangle$ for a system initialized in $|X\rangle=|\uparrow \downarrow\rangle$. Due to the symmetry relations for the correlation functions we have $C_{-X, X}(t)=1-C_{X X}(t)=1-C_{Y Y}(t)-C_{T_{0} S}(t)$ and thus find (using Eqs. (60) and (63) and neglecting terms of order $\left.\left(\sigma_{0}^{2}+x_{0}^{2}\right) / J^{2}\right)$,

$$
\begin{aligned}
& C_{-X, X}(t)=1-C_{X X}(t) \approx \frac{1}{2}-\frac{1}{2} e^{-\frac{1}{2}\left(\frac{t}{t_{0}^{\prime \prime}}\right)^{2}} \cos (|J| t), \\
& t_{0}^{\prime \prime}=\frac{|J|}{4 \sigma_{0}\left|x_{0}\right|}, \quad|J| \gg \max \left(\left|x_{0}\right|, \sigma_{0}\right), \quad t \ll t_{0}^{\prime}=\frac{|J|}{4 \sigma_{0}^{2}} .
\end{aligned}
$$

We obtain the maximum value for this correlator when $\tau_{s}=\frac{\pi}{|J|}$. The Gaussian has a decay time of $t_{0}^{\prime \prime}=\frac{|J|}{4 \sigma_{0}\left|x_{0}\right|}$, so for $x_{0} \rightarrow 0$ the Gaussian decay is negligible and we obtain unit fidelity for this SWAP operation $|\uparrow \downarrow\rangle \rightarrow|\downarrow \uparrow\rangle$ up to a global phase factor (which is not visible in the correlator).

From the SWAP operation it is only a small step towards the $\sqrt{\text { SWAP }}$ which we obtain when we let the system evolve with the same parameter values but for only half the time. Starting in the state $|X\rangle$ we obtain $|Y\rangle$ after applying a $\sqrt{\text { SWAP }}$. For large $|J|$ we find for the correlator $C_{Y X}$ in the limit $x_{0} \rightarrow 0$

$$
\begin{aligned}
& C_{Y X}(t)=\frac{1}{2}+\operatorname{sign}(J) \frac{1}{2} e^{-\frac{1}{2}\left(\frac{t}{t_{0}^{\prime \prime}}\right)^{2}} \sin (|J| t), \\
& t_{0}^{\prime \prime}=\frac{|J|}{4 \sigma_{0}\left|x_{0}\right|},|J| \gg \max \left(\left|x_{0}\right|, \sigma_{0}\right), t \ll t_{0}^{\prime}=\frac{|J|}{4 \sigma_{0}^{2}} .
\end{aligned}
$$

Here again the time scale of the Gaussian decay is $\frac{|J|}{4 \sigma_{0}\left|x_{0}\right|}$ and approaches infinity for $x_{0} \rightarrow 0$. The time during which we have to operate with these values of the parameters $J$ and $x_{0}$ is now $\tau_{s}=\frac{\pi}{2|J|}$. Our calculations show that for the time during which $J$ is pulsed high there is a regime in which unit fidelity may be approached. The reduced visibility in the experiment ${ }^{9}$ may be due to several reasons such as reduced visibility in the readout of $|\downarrow \uparrow\rangle$ or the initialization of $|\uparrow \downarrow\rangle$.

\section{CONCLUSION}

We have developed a method that uses the measurement of a Rabi resonance in the quantum-dot spin qubit to narrow the distribution of the nuclear spin states. This method relies on Rabi oscillations induced via an oscillation of the singlet-triplet splitting $J$ in the subspace $S^{z}=0$ of two electrons in a double quantum dot forming a two-qubit system. Further, we have calculated several correlators in the $S^{z}=0$ subspace for static $J$ and found that the transverse components of pseudo-spin have a slower decay than the longitudinal one. We have also discussed the implementation and fidelity of the $\sqrt{\mathrm{SWAP}}$ gate in this system and the initialization to the $|\uparrow \downarrow\rangle,|\downarrow \uparrow\rangle$ states.

\section{Acknowledgments}

We thank G. Burkard, M. Duckheim, J. Lehmann, F. H. L. Koppens, D. Stepanenko and, in particular, A. Yacoby for useful discussions. We acknowledge financial support from the Swiss NSF, the NCCR nanoscience, EU NoE MAGMANet, DARPA, ARO, ONR, JST ICORP, and NSERC of Canada.

\section{APPENDIX A: DRIFT IN $\delta h^{z}$}

In addition to spin diffusion, driven by the nuclear dipole-dipole interaction, there may also be a change in $\delta h^{z}$ due to corrections to the projected effective Hamiltonian considered here (see Ref. 18, Appendix B for details). After tracing out the electron pseudo-spin in state $\rho_{S}$, these correction terms give rise to an electronmediated nuclear spin-spin interaction which, in general, takes the form of an anisotropic (XYZ) Heisenberg interaction

$$
H_{n n}=\operatorname{Tr}_{S}\left\{\rho_{S} H\right\}=\sum_{i, j, \alpha=\{x, y, z\}} J_{i j}^{\alpha} I_{i}^{\alpha} I_{j}^{\alpha} .
$$

Here, the indices $i$ and $j$ run over all nuclear spin sites.

We use the corrections to leading order in the inverse Zeeman splitting $1 / \epsilon_{z}\left(\epsilon_{z}=g \mu_{B} B\right)$ given in Ref. 18. This gives the typical value of the exchange constants $\left|J_{i j}^{\alpha}\right| \sim A^{2} / N^{2} \epsilon_{z}$. Assuming an unpolarized nuclear spin state, each nuclear spin will therefore precess in an effective mean field generated by all other spins in the dot of typical magnitude

$$
h_{\mathrm{eff}} \sim \sqrt{N}\left|J_{i j}^{\alpha}\right| \sim A^{2} / N^{\frac{3}{2}} \epsilon_{z} .
$$

This effective field will result in precession of the nuclear spins about an arbitrary angle (and hence, may change the value of $\delta h^{z}$ ) on a time scale

$$
\tau_{p} \sim N^{\frac{3}{2}} \epsilon_{z} / A^{2} \sim 10^{-2} \mathrm{~s}
$$

where we have assumed $N=10^{6}$ nuclear spins within the quantum dot, and $\epsilon_{z} / g \mu_{B}=A / g \mu_{B} \simeq 3.5 \mathrm{~T}$ for the time estimate. This is only a worst-case estimate, which neglects the effects, e.g., of a Knight-shift gradient (due to strong confinement of the electron), which may further weaken the dynamical effect discussed here. We expect the dipolar nuclear spin diffusion time to be the limiting time scale for nuclear spin dynamics, in light of experiments on diffusion near donor impurities in GaAs. ${ }^{27}$ If the effect giving rise to $\tau_{p}$ in Eq. A3 were significant, it could be further suppressed by choosing a larger quantum 
dot size or stronger magnetic field, thus allowing many electron spin measurements on the time scale of variation of $\delta h^{z}$.

\section{APPENDIX B: MEASUREMENT}

In this appendix we describe how a single measurement of the two-electron system affects the nuclear spin state. We give the analytical expression for the diagonal elements of the nuclear spin density operator after a measurement.

At $t=0$ the system is described by the following density operator

$$
\rho(0)=\rho_{e}(0) \otimes \rho_{I}(0)=|+\rangle\left\langle+\left|\otimes \sum_{i} p_{i}\right| \psi_{I}^{i}\right\rangle\left\langle\psi_{I}^{i}\right|,
$$

with nuclear spin state $\left|\psi_{I}^{i}\right\rangle=\sum_{n} a_{n}^{i}|n\rangle$. The Hamiltonian $H_{0}$ of Eq.(1) acts on the the nuclear-spin system as $H_{0}|n\rangle=H_{n}|n\rangle$, where in $H_{n}$ the operator $\delta h^{z}$ has been replaced by $\delta h_{n}^{z}$ (because $\delta h^{z}|n\rangle=\delta h_{n}^{z}|n\rangle$ ). Since $\left[H_{0}, \delta h^{z}\right]=0$, only the diagonal elements of the nuclear density operator $\rho_{I}$ (in the basis of $\delta h^{z}$ ) enter in matrix elements for operators acting only on the twoelectron system. As described in Section $\amalg A$ these diagonal elements $\rho_{I}(n)=\rho_{I}(n, 0)=\left\langle n\left|\operatorname{Tr}_{e}\{\rho(0)\}\right| n\right\rangle$ describe a continuous Gaussian distribution in the continuum limit. The trace over the electron system is defined as $\operatorname{Tr}_{e} \rho(t)=\langle+|\rho(t)|+\rangle+\langle-|\rho(t)|-\rangle$ and for $\rho_{I}(n, 0)$ we have

$$
\rho_{I}(n, 0)=\sum_{i} p_{i}\left|a_{n}^{i}\right|^{2} .
$$

The time evolution operators $U(t)$ and $U_{n}(t)$ are defined through $i \dot{U}(t)=H_{0}(t) U(t)$ and $i \dot{U}_{n}(t)=H_{n}(t) U_{n}(t)$ and thus the density operator $\rho(0)$ evolves under the Hamiltonian $H_{0}$ as

$$
\begin{aligned}
\rho(t) & =U(t) \rho(0) U^{\dagger}(t) \\
& =U(t)\left(\rho_{e}(0) \otimes \sum_{i} \sum_{n, l} p_{i} a_{n}^{i} a_{l}^{i^{*}}|n\rangle\langle l|\right) U^{\dagger}(t) \\
& =\sum_{n, l}\left(U_{n}(t) \rho_{e}(0) U_{l}^{\dagger}(t) \otimes \sum_{i} p_{i} a_{n}^{i} a_{l}^{i^{*}}|n\rangle\langle l|\right) .
\end{aligned}
$$

At time $t_{m}$ a measurement in the basis of $|+\rangle$ and $|-\rangle$ is performed on one single two-electron system coupled to nuclear spins. Since the outcome of this measurement is known, the state of the system after the measurement is 43 (the result depends on whether $|+\rangle$ or $|-\rangle$ was measured)

$$
\begin{aligned}
\rho^{(1, \pm)}\left(t_{m}\right)= & \frac{| \pm\rangle\left\langle \pm\left|\rho\left(t_{m}\right)\right| \pm\right\rangle\langle \pm|}{P^{ \pm}\left(t_{m}\right)} \\
= & \sum_{n, l}\left(| \pm\rangle\left\langle \pm\left|U_{n}\left(t_{m}\right) \rho_{e}(0) U_{l}^{\dagger}\left(t_{m}\right)\right| \pm\right\rangle\langle \pm|\right. \\
& \left.\otimes \sum_{i} p_{i} a_{n}^{i} a_{l}^{i *}|n\rangle\langle l|\right) \frac{1}{P^{ \pm}\left(t_{m}\right)},
\end{aligned}
$$

with

$$
\begin{aligned}
P^{ \pm}\left(t_{m}\right) & =\operatorname{Tr}_{I} \operatorname{Tr}_{e}\left\{| \pm\rangle\langle \pm| \rho\left(t_{m}\right)\right\} \\
& =\sum_{i} \sum_{n} \frac{1}{2}\left(1 \pm\left\langle\tau^{z}\left(t_{m}\right)\right\rangle_{n}\right) p_{i}\left|a_{n}^{i}\right|^{2}
\end{aligned}
$$

where $\operatorname{Tr}_{I} A=\sum_{n}\langle n|A| n\rangle$ and $\left\langle\tau^{z}(t)\right\rangle_{n}$ is given in Eq. (44). Here, $P^{ \pm}\left(t_{m}\right)$ is the probability to measure $| \pm\rangle$ at time $t_{m}$. We are mainly interested in the diagonal elements of the nuclear density operator $\rho_{I}$ after the measurement.

$$
\begin{aligned}
\rho_{I}^{(1, \pm)}\left(n, t_{m}\right) & =\left\langle n\left|\operatorname{Tr}_{e} \rho^{(1, \pm)}\left(t_{m}\right)\right| n\right\rangle \\
& =\frac{\rho_{I}(n, 0)}{P^{ \pm}\left(t_{m}\right)}\left\langle \pm\left|U_{n}\left(t_{m}\right) \rho_{e}(0) U_{n}^{\dagger}\left(t_{m}\right)\right| \pm\right\rangle \\
& =\frac{\rho_{I}(n, 0)}{P^{ \pm}\left(t_{m}\right)} \frac{1}{2}\left(1 \pm\left\langle\tau^{z}\left(t_{m}\right)\right\rangle_{n}\right) .
\end{aligned}
$$

Using Eq. (41) we find

$$
\begin{aligned}
\rho_{I}^{(1,+)}\left(n, t_{m}\right)= & \frac{\rho_{I}(n, 0)}{P^{+}\left(t_{m}\right)} \frac{1}{2}\left(\frac{2\left(\Omega_{n}-\omega\right)^{2}}{\left(\Omega_{n}-\omega\right)^{2}+(j / 2)^{2}}\right. \\
& \left.+\frac{(j / 2)^{2}\left(1+\cos \left(\omega^{\prime} t_{m}\right)\right)}{\left(\Omega_{n}-\omega\right)^{2}+(j / 2)^{2}}\right)
\end{aligned}
$$

and

$$
\rho_{I}^{(1,-)}\left(n, t_{m}\right)=\frac{\rho_{I}(n, 0)}{P^{-}\left(t_{m}\right)} \frac{1}{2} \frac{(j / 2)^{2}\left(1-\cos \left(\omega^{\prime} t_{m}\right)\right)}{\left(\Omega_{n}-\omega\right)^{2}+(j / 2)^{2}},
$$

where $\omega^{\prime}$ is given in Eq. (15) and depends on the eigenvalue $\delta h_{n}^{z}$ of the nuclear spin eigenstate through $\Omega_{n}$.

Parenthetically, we note that in the case (not described in this article) where the measurement is performed on an ensemble of many different double quantum dots, the state of the ensemble after the measurement is $\underline{44}$

$$
\begin{aligned}
\rho_{\text {ens }}^{(1)}\left(t_{m}\right)= & \sum_{n, l}\left(|+\rangle\left\langle+\left|U_{n}\left(t_{m}\right) \rho_{e}(0) U_{l}^{\dagger}\left(t_{m}\right)\right|+\right\rangle\langle+|\right. \\
& \left.+|-\rangle\left\langle-\left|U_{n}\left(t_{m}\right) \rho_{e}(0) U_{l}^{\dagger}\left(t_{m}\right)\right|+\right\rangle\langle+|\right) \\
& \otimes \sum_{i} p_{i} a_{n}^{i} a_{l}^{i *}|n\rangle\langle l|,
\end{aligned}
$$

and the nuclear-spin distribution has not changed. If a complete measurement of the Rabi-resonance lineshape would be performed on an ensemble of double dots, the result would be the Voigt profile described in Sec. ЩA 
1 D. Loss and D. P. DiVincenzo, Phys. Rev. A 57, 120 (1998).

2 H.-A. Engel and D. Loss, Science 309, 586 (2005).

3 V. Cerletti, W. A. Coish, O. Gywat, and D. Loss, Nanotechnology 16, 27 (2005).

${ }^{4}$ K. Ono, D. G. Austing, Y. Tokura, and S. Tarucha, Science 297, 1313 (2002).

${ }^{5}$ K. Ono and S. Tarucha, Phys. Rev. Lett. 92, 256803 (2004)

6 A. C. Johnson, J. R. Petta, J. M. Taylor, A. Yacoby, M. D. Lukin, C. M. Marcus, M. P. Hanson, and A. C. Gossard, Nature 435, 925 (2005).

7 T. Hatano, M. Stopa, and S. Tarucha, Science 309, 268 (2005).

8 F. H. L. Koppens, J. A. Folk, J. M. Elzerman, R. Hanson, L. H. W. van Beveren, I. T. Vink, H. P. Tranitz, W. Wegscheider, L. P. Kouwenhoven, and L. M. K. Vandersypen, Science 309, 1346 (2005).

9 J. R. Petta, A. C. Johnson, J. M. Taylor, E. A. Laird, A. Yacoby, M. D. Lukin, C. M. Marcus, M. P. Hanson, and A. C. Gossard, Science 309, 2180 (2005).

10 J. Schliemann, A. Khaetskii, and D. Loss, J. Phys.: Condens. Matter 15, 1809 (2003).

11 G. Burkard, D. Loss, and D. P. DiVincenzo, Phys. Rev. B 59, 2070 (1999).

12 S. I. Erlingsson, Y. V. Nazarov, and V. I. Fal'ko, Phys. Rev. B 64, 195306 (2001).

13 S. I. Erlingsson and Y. V. Nazarov, Phys. Rev. B 66, 155327 (2002).

14 A. V. Khaetskii, D. Loss, and L. Glazman, Phys. Rev. Lett. 88, 186802 (2002).

15 I. A. Merkulov, A. L. Efros, and M. Rosen, Phys. Rev. B 65, 205309 (2002).

16 A. Khaetskii, D. Loss, and L. Glazman, Phys. Rev. B 67, 195329 (2003).

17 W. A. Coish and D. Loss, Phys. Rev. B 70, 195340 (2004).

18 W. A. Coish and D. Loss, Phys. Rev. B 72, 125337 (2005).

19 A. S. Bracker, E. A. Stinaff, D. Gammon, M. E. Ware, J. G. Tischler, A. Shabaev, A. L. Efros, D. Park, D. Gershoni, V. L. Korenev, et al., Phys. Rev. Lett. 94, 047402 (2005).

20 M. V. Dutt, J. Cheng, B. Li, X. Xu, X. Li, P. R. Berman, D. G. Steel, A. S. Bracker, D. Gammon, S. E. Economou, et al., Phys. Rev. Lett. 94, 227403 (2005).

21 D. Paget, G. Lampel, B. Sapoval, and V. I. Safarov, Phys. Rev. B 15, 5780 (1977).

22 R. de Sousa and S. Das Sarma, Phys. Rev. B 67, 033301 (2003).

${ }^{23}$ N. Shenvi, R. de Sousa, and K. B. Whaley, Phys. Rev. B 71, 224411 (2005).

24 W. Yao, R.-B. Liu, and L. J. Sham, ArXiv Condensed Matter e-prints (2005), arXiv:cond-mat/0508441.

25 A. M. Steane, Phys. Rev. A 68, 042322 (2003).

26 A. Imamoğlu, E. Knill, L. Tian, and P. Zoller, Phys. Rev. Lett. 91, 017402 (2003).
27 D. Paget, Phys. Rev. B 25, 4444 (1982).

28 Y. Kato, R. C. Myers, D. C. Driscoll, A. C. Gossard, J. Levy, and D. D. Awschalom, Science 299, 1201 (2003).

29 G. Giedke, J. M. Taylor, D. D'Alessandro, M. D. Lukin, and A. Imamoğlu, ArXiv Quantum Physics e-prints (2005), arXiv:quant-ph/0508144.

30 D. Stepanenko, G. Burkard, G. Giedke, and A. Imamoğlu, ArXiv Condensed Matter e-prints (2005), arXiv:condmat/0512362.

31 J. Levy, Phys. Rev. Lett. 89, 147902 (2002).

32 C. P. Slichter, Principles of Magnetic Resonance (Springer Verlag, Berlin, 1980).

33 B. H. Armstrong, J. Quant. Spectrosc. Radiat. Transfer 7, 61 (1967).

34 A. M. Stoneham, J. Phys. D: Appl. Phys. 5, 670 (1972).

35 P. Minguzzi and A. di Lieto, J. Mol. Spectrosc. 109, 388 (1985).

${ }^{36}$ H.-A. Engel, V. N. Golovach, D. Loss, L. M. K. Vandersypen, J. M. Elzerman, R. Hanson, and L. P. Kouwenhoven, Phys. Rev. Lett. 93, 106804 (2004).

37 By "low time resolution", we mean that the measurement is performed at an unknown time $t_{m}$ (giving rise to state $\left.\rho\left(t_{m}\right)\right)$ in the interval $\Delta t=t_{b}-t_{a}$, with a uniform probability density $1 / \Delta t$. The state after the measurement is then $\frac{1}{\Delta t} \int_{t_{a}}^{t_{b}} d t_{m} \rho\left(t_{m}\right)$.

38 This assumption is not necessary for our narrowing scheme. However, it does allow for the derivation of the analytical formulas in this section, which give insight into the mechanism of narrowing. In the case of perfect time resolution, one would have an additional factor of $\left(1-\cos \left(\omega^{\prime} t_{m}\right)\right)$ in Eq. (29) ( $\omega^{\prime}$ is given in Eq. (5) ) and would also have to take into account the time $t_{m}$ at which each measurement was performed.

39 We have performed more than 60 runs of the simulation, varying $M$ and $j / \sigma_{0}$.

40 M. Borhani, V. N. Golovach, and D. Loss, ArXiv Condensed Matter e-prints (2005), arXiv:cond-mat/0510758.

41 A. Barenco, C. H. Bennett, R. Cleve, D. P. DiVincenzo, N. Margolus, P. Shor, T. Sleator, J. A. Smolin, and H. Weinfurter, Phys. Rev. A 52, 3457 (1995).

42 R. Requist, J. Schliemann, A. G. Abanov, and D. Loss, Phys. Rev. B 71, 115315 (2005).

43 The measurement performed at $t_{m}$ is not sufficient to distinguish the different states $\left|\psi_{I}^{i}\right\rangle$. This is because it is a measurement in the two-electron system and it only has an effect on the nuclear spin system in a way that some eigenstates $|n\rangle$ gain weight and some loose. This happens for all states $\left|\psi_{I}^{i}\right\rangle$ in the same way and thus from such a measurement we cannot tell into which of the states $\left|\psi_{I}^{i}\right\rangle$ the system has collapsed.

44 A. Peres, Quantum Theory: Concepts and Methods (Kluwer Academic Publishers, 1993). 